\title{
Well-posedness of Semilinear Heat Equations in $L^{1}$
}

\author{
R. Laister ${ }^{a, *}$, M. Sierżęga ${ }^{b}$ \\ ${ }^{a}$ Department of Engineering Design and Mathematics, \\ University of the West of England, Bristol BS16 1QY, UK. \\ ${ }^{b}$ Faculty of Mathematics, Informatics and Mechanics, University of Warsaw, \\ Banacha 2, 02-097 Warsaw, Poland.
}

\begin{abstract}
The problem of obtaining necessary and sufficient conditions for local existence of nonnegative solutions in Lebesgue spaces for semilinear heat equations having monotonically increasing source term $f$ has only recently been resolved (Laister et al. (2016)). There, for the more difficult case of initial data in $L^{1}$, a necessary and sufficient integral condition on $f$ emerged. Here, subject to this integral condition, we consider other fundamental properties of solutions with $L^{1}$ initial data of indefinite sign, namely: uniqueness, regularity, continuous dependence and comparison. We also establish sufficient conditions for the global-in-time continuation of solutions for small initial data in $L^{1}$.
\end{abstract}

Keywords: heat equation, existence, uniqueness, continuous dependence, comparison, global solution.

\section{Introduction}

In this paper we address fundamental questions concerning the well-posedness of semilinear heat equations of the form

$$
u_{t}=\Delta u+f(u), \quad u(0)=\phi,
$$

where $f: \mathbb{R} \rightarrow \mathbb{R}$ is locally Lipschitz continuous and non-decreasing, $f(0)=0$ and the initial condition $\phi$ is taken in the space $L^{1}\left(\mathbb{R}^{n}\right)$. In a previous work [13] we established

\footnotetext{
* Corresponding author

Email addresses: Robert.Laister@uwe.ac.uk (R. Laister), M.Sierzega@mimuw.edu.pl (M. Sierżęga)
} 
sharp results on the local existence of non-negative solutions when $f:[0, \infty) \rightarrow[0, \infty)$ and $\phi \in L_{+}^{1}\left(\mathbb{R}^{n}\right)$. Here we consider issues of existence and uniqueness for initial data of indefinite sign, thus providing a more comprehensive account of the well-posedness problem for (1.1). Furthermore we obtain results on the regularity, continuous dependence, comparison and global existence of solutions.

The special case of the homogeneous power law nonlinearity $f(u)=|u|^{p-1} u$ in (1.1), commonly referred to as the Fujita equation, has attracted much attention. In fact, the original spark of interest in this restricted setting [8] has inspired the bulk of subsequent developments. The interested reader is invited to consult [19] and the extensive list of references therein for a detailed account of the state of the art for this problem.

A particularly important avenue of research concerns local well-posedness for singular (unbounded) initial data and power-like nonlinearities satisfying

$$
|f(u)-f(v)| \leq C\left(1+|u|^{p-1}+|v|^{p-1}\right)|u-v|, \quad p>1 .
$$

Sufficient conditions for local existence in Lebesgue spaces for classes of nonlinearities of this type were established via a contraction mapping argument in [22, 23] (see also [3]). There the interplay between the power-like nonlinearity and Lebesgue norms produced a clear-cut characterisation of local existence in $L^{q}$ with respect to a particular exponent $q^{*}=\frac{n(p-1)}{2}$. Thus when $q \geq 1$ and $q>q^{*}$, or $q=q^{*}>1$, then for every $\phi \in L^{q}$ there exists a $T_{\phi}>0$ and a solution in $C\left(\left[0, T_{\phi}\right], L^{q}\right) \cap L_{\text {loc }}^{\infty}\left(\left(0, T_{\phi}\right), L^{\infty}\right)$, i.e. a classical $L^{q}$-solution. The special case of the pure power law $f(u)=|u|^{p-1} u$ shows that $q^{*}$ is indeed a critical value as the problem can exhibit non-existence of non-negative solutions whenever $1 \leq q<q^{*}$ [23, Theorem 5]. Lastly, the end-point case $q=q^{*}=1$ differs from the critical regime for $q_{*}>1$ in that there are non-negative data for which no non-negative solution may be defined [3, Theorem 11] and [4].

Uniqueness results were also established in [23], but in a restricted class of functions where solutions satisfy a certain growth bound as $t \rightarrow 0$ a priori. This growth restriction was removed in [3], providing a stronger uniqueness result in the space of classical $L^{q}$ solutions. Hitherto these were the best results available regarding local well-posedness of 
(1.1) in $L^{q}$.

For non-power-like nonlinearities this tight correspondence between the source term growth and integrability of the initial data generally fails and two problems arise:

(A) given $f$, characterise the set $X$ of initial data for which (1.1) has a solution;

(B) given the set $X$ of initial data, characterise those sources $f$ for which (1.1) has a solution for every initial datum in $X$.

These two problems are of course subject to a variety of further ramifications, e.g.,we need to specify the solution concept and then we may insist that $X$ is a Banach space say, or that $f$ is a smooth function, etc.

One definitive result in direction (A), concerning non-negative solutions of the Fujita equation on bounded domains with Dirichlet boundary conditions, was given in [2]. With regards to problem (B), in [13] we gave a necessary and sufficient condition for a nonnegative, non-decreasing, continuous function $f$ to give rise to a local solution bounded in $L_{+}^{q}\left(\mathbb{R}^{n}\right)$ for all initial data in $L_{+}^{q}\left(\mathbb{R}^{n}\right)$. Specifically, for $q>1$, and assuming $f$ to be Lipschitz continuous at zero, local existence holds for all $\phi \in L_{+}^{q}\left(\mathbb{R}^{n}\right)$ if and only if

$$
\limsup _{u \rightarrow \infty} \frac{f(u)}{u^{1+2 q / n}}<\infty \text {. }
$$

Morally, this result means that the power-like nonlinearities of (1.2) are essentially the whole story with regards to local existence in $L_{+}^{q}\left(\mathbb{R}^{n}\right)$ when $q>1$, i.e. it is precisely those nonlinearities which are majorised by $u^{1+2 q / n}$ which are compatible with Lebesgue spaces of non-negative initial data. However, power-like nonlinearities certainly do not tell the whole story in the more delicate case $q=1$. For then the condition for existence

$$
|f(u)-f(v)| \leq C\left(1+|u|^{p-1}+|v|^{p-1}\right)|u-v|, \quad p<1+2 / n
$$

in 3, 23] is sufficient but not necessary. In fact the optimal ('critical') condition obtained in [13] for the local existence property in $L_{+}^{1}\left(\mathbb{R}^{n}\right)$ reads

$$
\int_{1}^{\infty} s^{-(1+2 / n)} \ell_{+}(s) \mathrm{d} s<\infty
$$


where

$$
\ell_{+}(s)=\sup _{0<t \leq s} \frac{f(t)}{t}
$$

(Note that $\ell_{+}(s)$ was denoted by $F(s)$ in [13].) Thus there are nonlinearities $f$ which satisfy the integral condition (1.4) but which do not satisfy (1.3). See [13, Section 4.4] for such an example in the bounded domain case, but which also applies to the whole space $\mathbb{R}^{n}$. We see again that $L^{1}$ differs qualitatively from higher Lebesgue spaces and concepts like 'critical nonlinearity' require careful treatment.

It is worth mentioning that the contraction mapping arguments in [3, 22, 23] do not require monotonicity of $f$. Whilst we obtain stronger results in terms of the growth of $f$ at infinity, our methods are reliant upon monotonicity. For some alternative, related results see also the recent developments in [7].

It is natural then to ask whether the gap between the existence conditions (1.3) and (1.4) might lead to improvements of current uniqueness results when $q=1$, namely [ $[3$, Theorem 1]. Indeed, we show in Theorem 2.4 that this is the case. Whereas uniqueness of classical $L^{1}$-solutions is known to hold under (1.3), we are able to weaken this condition accordingly; see hypothesis (I2) in Section 2.3. There we adapt some of the methods of [3] but without recourse to the contraction mapping theorem. It should be noted however, that our choice of working with classical solutions (for $t>0$ ) with initial data in $L^{1}\left(\mathbb{R}^{n}\right)$ is instrumental in uniqueness considerations; non-uniqueness can result otherwise [15, 17]. Furthermore our monotone methods yield additional results on continuous dependence and comparison in this more general setting, see Theorem 2.6, which extend further those of [3].

We go on to consider some special classes of source terms and initial data. For example, when $f$ is convex on $[0, \infty)$ and odd then the integral condition for existence (namely (I1) of Section 2.2, similar in spirit to that of (1.4)) is equivalent to the one for both existence and uniqueness (namely (I2)) - see Corollary 3.2. Specialising further to the case of non-negative ititial data then leads to a sharp (necessary and sufficient) result, Corollary [3.5, regarding local well-posedness of (1.1) within the class of convex source terms on $[0, \infty)$ and initial data in $L_{+}^{1}\left(\mathbb{R}^{n}\right)$. 
In the context of global solutions it has long been known for convex sources $f$ satisfying the ODE blow-up criterion $\int^{\infty} \mathrm{d} s / f(s)<\infty$, that blow-up in (1.1) can occur for large initial data and that small initial data can evolve into global-in-time solutions, see [11]. In the seminal paper [8] Fujita obtained an important result for the special case of $f(u)=u^{p}$ on the whole space, whereby the critical exponent $p_{F}:=1+2 / n$ separated two regimes: for $1<p<p_{F}$ every non-negative, non-trivial classical solution blows up in finite time, whereas for $p>p_{F}$ it is possible to find small initial conditions evolving into global-in-time solutions. The so-called 'critical case' $p=p_{F}$ was later shown to be in the blow-up regime [10, 21]. Notions of smallness abound, but Fujita's original approach involved data bounded above by a small Gaussian. Subsequent developments defined smallness via a norm in an appropriate Banach space. The homogeneous power nonlinearity $f(u)=|u|^{p-1} u$ is naturally compatible with the Lebesgue space structure and in [24] Weissler succeeded in replacing the small Gaussian bound with a small critical Lebesgue norm $\|\cdot\|_{p_{F}}$. In this paper too we establish some global existence results for initial data with sufficiently small $L^{1}$-norm, see Theorems 2.7 and 2.8 .

Critical and supercritical phenomena appear often in semilinear heat equations when the averaging action of diffusion and the amplifying action of the nonlinear term exert a comparable infulence on the behaviour of the solution. The character of this inteplay may lead to subtle effects in the dynamics of singularity formation [6], existence of nonsmooth solutions [18] and asymptotic behaviour of global solutions [5]. In the context of power law source terms, a considerable body of work on these topics has been developed over the past three decades (see [19] for an overview). In this work we have investigated the critical balance with regards to the question of local existence of solutions for source terms in a more general class of functions. Whether similar generalisations are possible in the blow-up theory and threshold behaviour of global solutions remains to be seen and poses interesting research challenges for the future. 


\section{Well-Posedness in $L^{1}$}

\subsection{Preliminaries}

Throughout we use $p_{F}$ to denote the Fujita exponent $p_{F}=1+2 / n$. We write $\|\cdot\|_{q}$ for the norm in $L^{q}\left(\mathbb{R}^{n}\right)$ and denote by $\{S(t)\}_{t \geq 0}$ the heat semigroup on $L^{q}\left(\mathbb{R}^{n}\right)(q \geq 1)$ generated by $-\Delta$ on $\mathbb{R}^{n}$ with the explicit representation formula

$$
[S(t) \phi](x)=\int_{\mathbb{R}^{n}} G(x-y, t) \phi(y) \mathrm{d} y, \quad \phi \in L^{q}\left(\mathbb{R}^{n}\right),
$$

where $G$ is the Gaussian heat kernel

$$
G(x, t)=(4 \pi t)^{-n / 2} \mathrm{e}^{-|x|^{2} / 4 t} .
$$

It is well-known that $S(t)$ is a $C_{0}$-semigroup for all $q \geq 1$ finite.

As is commonplace for semilinear problems we will study solutions of (1.1) via the variation of constants formula

$$
u(t)=\mathscr{F}(u ; \phi):=S(t) \phi+\int_{0}^{t} S(t-s) f(u(s)) \mathrm{d} s .
$$

We now make precise our solution concepts (see e.g., [19, Section 15]), setting $Q_{T}=\mathbb{R}^{n} \times$ $(0, T)$.

\section{Definition 2.1.}

(i) We say that $u$ is a classical $L^{1}$-solution of (1.1) on $[0, T)$ if $u$ satisfies (1.1) in the classical sense in $Q_{T}, u \in C\left([0, T), L^{1}\left(\mathbb{R}^{n}\right)\right) \cap L_{\text {loc }}^{\infty}\left((0, T), L^{\infty}\left(\mathbb{R}^{n}\right)\right)$ and $u(0)=\phi$.

(ii) We say that (1.1) is well-posed in $L^{1}\left(\mathbb{R}^{n}\right)$ if for all $\phi \in L^{1}\left(\mathbb{R}^{n}\right)$ there exists $T=T(\phi)>0$ and a unique classical $L^{1}$-solution of (1.1) on $[0, T)$.

Definition 2.2. Let $T>0$. We say that a measurable, finite almost everywhere (a.e.) function $w: Q_{T} \rightarrow \mathbb{R}$ is an integral supersolution (respectively subsolution) of (1.1) on $Q_{T}$ if $w$ satisfies $\mathscr{F}(w ; \phi) \leq w$ (respectively $\mathscr{F}(w ; \phi) \geq w)$ a.e. in $Q_{T}$, with $\mathscr{F}$ as in (2.1). If $w$ is both an integral supersolution and an integral subsolution of (1.1) on $Q_{T}$ then we say that $w$ is an integral solution of (1.1) on $Q_{T}$. 
In what follows we will often write 'supersolution' instead of 'integral supersolution' whenever the context is clear, and likewise for 'subsolution'.

We recall the following standard smoothing estimate for the heat semigroup when $1 \leq$ $q \leq r \leq \infty$ and $\phi \in L^{q}\left(\mathbb{R}^{n}\right)$ (see e.g., [3, Lemma 7] or [19, Proposition 48.4]):

$$
\|S(t) \phi\|_{r} \leq t^{-\frac{n}{2}\left(\frac{1}{q}-\frac{1}{r}\right)}\|\phi\|_{q}, \quad t>0 .
$$

The following lemma is a minor adaptation of [3, Lemma 8] for bounded domains to the whole space. Here we make use of the fact that $S(t)$ is a $C_{0}$-semigroup to offer a slightly different proof to the one given there.

Lemma 2.1. Let $1 \leq q<r \leq \infty$ and $\alpha=\frac{n}{2}\left(\frac{1}{q}-\frac{1}{r}\right)$. If $\phi \in L^{q}\left(\mathbb{R}^{n}\right)$ then

$$
\lim _{t \rightarrow 0} t^{\alpha}\|S(t) \phi\|_{r}=0 \text {. }
$$

Proof. For any $\varepsilon>0$,

$$
\begin{aligned}
t^{\alpha}\|S(t) \phi\|_{r} & \leq t^{\alpha}\|S(t)(\phi-S(\varepsilon) \phi)\|_{r}+t^{\alpha}\|S(\varepsilon) S(t) \phi\|_{r} \\
& \leq\|\phi-S(\varepsilon) \phi\|_{q}+t^{\alpha} \varepsilon^{-\alpha}\|S(t) \phi\|_{q} \\
& \leq\|\phi-S(\varepsilon) \phi\|_{q}+t^{\alpha} \varepsilon^{-\alpha}\|\phi\|_{q}
\end{aligned}
$$

so that

$$
\lim _{t \rightarrow 0} t^{\alpha}\|S(t) \phi\|_{r} \leq\|\phi-S(\varepsilon) \phi\|_{q} .
$$

Letting $\varepsilon \rightarrow 0$ and using the strong continuity of $S(\cdot)$ for $q \in[1, \infty)$, yields the result.

Remark 2.1. The two ingredients needed in the proof of Lemma 2.1, namely the smoothing estimate and the strong continuity of the semigroup, may be generalised to the setting of fractional power spaces $X^{\alpha}, \alpha \geq 0$, associated with a sectorial operator $A$, such that $A: D(A) \subset X^{0} \rightarrow X^{0}$, where $X^{0}$ is a Banach space. Then $\{S(t)\}_{t \geq 0}$ is an analytic semigroup generated by $A$ which, in particular, satisfies the bounds

$$
\|S(t) \phi\|_{X^{\alpha}} \leq M\|\phi\|_{X^{\alpha}}, \quad\|S(t) \phi\|_{X^{\alpha}} \leq M t^{-\alpha}\|\phi\|_{X^{0}}, \quad t>0,
$$

for some constant $M>0$. See e.g., [1] and the references therein. 


\subsection{Local Existence}

We introduce the following monotonicity hypothesis for $f$ :

(M) $\quad f: \mathbb{R} \rightarrow \mathbb{R}$ is locally Lipschitz continuous, non-decreasing and $f(0)=0$.

For $f$ satisfying $(\mathrm{M})$ we may define the non-decreasing function $\ell:[0, \infty) \rightarrow[0, \infty)$ by

$$
\ell(s)=\sup _{0<|t| \leq s} \frac{f(t)}{t} \quad(s>0), \quad \ell(0)=0 .
$$

Observe that by (M) and taking $t=s$ in (2.3), we have

$$
f(s) \leq s \ell(s), \quad s \geq 0
$$

and (taking $t=-s$ ),

$$
f(s) \geq s \ell(-s), \quad s \leq 0 .
$$

We now consider the issue of existence of solutions of (1.1), for which we introduce our second hypothesis:

$$
\text { (I1) } \int_{1}^{\infty} s^{-p_{F}} \ell(s) \mathrm{d} s<\infty
$$

where $p_{F}=1+2 / n$. Observe that (I1) is equivalent to

$$
\int_{0}^{1} \ell\left(\tau^{-n / 2}\right) \mathrm{d} \tau<\infty
$$

Theorem 2.2. (Uniform Existence.) Suppose $f$ satisfies (M) and (I1) and $B$ is any bounded subset of $L^{1}\left(\mathbb{R}^{n}\right)$. Then there exists a $T_{B}>0$ such that for all $\phi \in B$ there exist classical $L^{1}$-solutions $\underline{u}(t ; \phi)$ and $\bar{u}(t ; \phi)$ of $(1.1)$ with $\underline{u}(t ; \phi) \leq \bar{u}(t ; \phi)$ on $\left[0, T_{B}\right)$. Furthermore, for all $\phi \in B$,

$$
\lim _{t \rightarrow 0} t^{n / 2}\|\underline{u}(t ; \phi)\|_{\infty}=\lim _{t \rightarrow 0} t^{n / 2}\|\bar{u}(t ; \phi)\|_{\infty}=0
$$

Proof. For non-negative $\phi$, local existence was proved in $[13$, Theorem 5.1 (ii)] (see also [13, Theorem 4.4]). We adapt that proof here to obtain solutions for initial data of indefinite sign, 
on a uniform interval of existence with respect to $B$. We will show that there exists a $T_{B}>0$ such that for any $\phi \in B$ there is an integral supersolution $w(t)$ and an integral subsolution $v(t)$ with $v \leq w$. From this sub-supersolution pair we will then construct iteratively the solutions $\underline{u}$ and $\bar{u}$.

Let

$$
\phi^{-}=\min \{\phi, 0\} \leq 0, \quad \phi^{+}=\max \{\phi, 0\} \geq 0
$$

and for $t \geq 0$ set

$$
v(t)=2 S(t) \phi^{-} \leq 0, \quad w(t)=2 S(t) \phi^{+} \geq 0 .
$$

Clearly $v \leq 0 \leq w$ by monotonicity of $S(t)$ and the ordering $\phi^{-} \leq 0 \leq \phi^{+}$.

As $B$ is bounded there is a constant $K>0$ such that $\|\phi\|_{1} \leq K$ for all $\phi \in B$. Hence since $w \geq 0, \ell$ is non-decreasing and recalling (2.4),

$$
\begin{aligned}
S(t-s) f(w(s)) & =S(t-s) f\left(2 S(s) \phi^{+}\right) \leq S(t-s) \ell\left(2 S(s) \phi^{+}\right) 2 S(s) \phi^{+} \\
& \leq S(t-s) \ell\left(\left\|2 S(s) \phi^{+}\right\|_{\infty}\right) 2 S(s) \phi^{+} \leq \ell\left(2\left\|\phi^{+}\right\|_{1} s^{-n / 2}\right) 2 S(t) \phi^{+} \\
& \leq \ell\left(2\|\phi\|_{1} s^{-n / 2}\right) w(t) \leq \ell\left(2 K s^{-n / 2}\right) w(t)
\end{aligned}
$$

and so

$$
\begin{aligned}
\mathscr{F}(w ; \phi) & =S(t) \phi+\int_{0}^{t} S(t-s) f(w(s)) \mathrm{d} s \\
& \leq S(t) \phi^{+}+\left(\int_{0}^{t} \ell\left(2 K s^{-n / 2}\right) \mathrm{d} s\right) w(t) \\
& =\left(1 / 2+(2 K)^{2 / n} \int_{0}^{t(2 K)^{-2 / n}} \ell\left(\tau^{-n / 2}\right) \mathrm{d} \tau\right) w(t) .
\end{aligned}
$$

Consequently $\mathscr{F}(w ; \phi) \leq w$ whenever

$$
w(t)(g(t)-1 / 2) \leq 0,
$$

where $g$ is given by

$$
g(t)=(2 K)^{2 / n} \int_{0}^{t(2 K)^{-2 / n}} \ell\left(\tau^{-n / 2}\right) \mathrm{d} \tau
$$


Due to (2.6) we can choose $T_{B}>0$ such that $g(t) \leq \frac{1}{2}$ for all $t \in\left[0, T_{B}\right]$, i.e. $\mathscr{F}(w ; \phi) \leq w$ on $\left[0, T_{B}\right]$. Note in particular that the choice of $T_{B}$ depends only upon $K$, and hence $B$, but not upon $\phi$.

Now recall (2.5) and that $v \leq 0$. Setting $\hat{\phi}=-\phi^{-} \geq 0$ we obtain

$$
\begin{aligned}
S(t-s) f(v(s)) & =S(t-s) f\left(2 S(s) \phi^{-}\right) \geq S(t-s) \ell(2 S(s) \hat{\phi}) 2 S(s) \phi^{-} \\
& \geq S(t-s) \ell\left(\|2 S(s) \hat{\phi}\|_{\infty}\right) 2 S(s) \phi^{-} \geq \ell\left(2\|\hat{\phi}\|_{1} s^{-n / 2}\right) 2 S(t) \phi^{-} \\
& \geq \ell\left(2\|\phi\|_{1} s^{-n / 2}\right) v(t) \geq \ell\left(2 K s^{-n / 2}\right) v(t),
\end{aligned}
$$

so that

$$
\begin{aligned}
\mathscr{F}(v ; \phi) & =S(t) \phi+\int_{0}^{t} S(t-s) f(v(s)) \mathrm{d} s \\
& \geq S(t) \phi^{-}+\left(\int_{0}^{t} \ell\left(2 K s^{-n / 2}\right) \mathrm{d} s\right) v(t) \\
& =\left(1 / 2+\int_{0}^{t} \ell\left(2 K s^{-n / 2}\right) \mathrm{d} s\right) v(t) \\
& =(1 / 2+g(t)) v(t),
\end{aligned}
$$

recalling (2.7). Thus, $\mathscr{F}(v ; \phi) \geq v$ provided that

$$
v(t)(g(t)-1 / 2) \geq 0
$$

which evidently holds for all $t \in\left[0, T_{B}\right]$, as for $w$ above.

For notational convenience we now set $T=T_{B}$ in the remainder of the proof. For any $\phi \in B$ and $t \in[0, T]$, one may then use $v$ and $w$ to construct iteratively a decreasing sequence $w_{k}(t ; \phi)$ and an increasing sequence $v_{k}(t ; \phi)$ via the relations

$$
w_{k+1}(t ; \phi)=\mathscr{F}\left(w_{k} ; \phi\right), \quad w_{0}(t ; \phi)=w(t)
$$

and

$$
v_{k+1}(t ; \phi)=\mathscr{F}\left(v_{k} ; \phi\right), \quad v_{0}(t ; \phi)=v(t) .
$$

Using the monotonicity of $\mathscr{F}$ (see, for example, [20, Theorem 1] or [24, Theorem 1]) it is easy to verify by induction that for all $k$

$$
v \leq v_{k} \leq v_{k+1} \leq w_{k+1} \leq w_{k} \leq w
$$


Clearly therefore, the pointwise limits

$$
\lim _{k \rightarrow \infty} v_{k}(x, t ; \phi)=: \underline{u}(x, t ; \phi), \quad \lim _{k \rightarrow \infty} w_{k}(x, t ; \phi)=: \bar{u}(x, t ; \phi)
$$

exist and satisfy $v \leq \underline{u} \leq \bar{u} \leq w$ a.e. in $Q_{T}$. Furthermore, $v, w \in L^{1}\left(Q_{T}\right)$ since

$$
\begin{aligned}
\int_{Q_{T}}|w(x, t)| \mathrm{d} x \mathrm{~d} t & =\int_{0}^{T} \int_{\mathbb{R}^{n}} 2\left[S(t) \phi^{+}\right](x) \mathrm{d} x \mathrm{~d} t \leq 2 T\|\phi\|_{1}, \\
\int_{Q_{T}}|v(x, t)| \mathrm{d} x \mathrm{~d} t & =\int_{0}^{T} \int_{\mathbb{R}^{n}}-2\left[S(t) \phi^{-}\right](x) \mathrm{d} x \mathrm{~d} t \leq 2 T\|\phi\|_{1} .
\end{aligned}
$$

Clearly, both $|\underline{u}|$ and $|\bar{u}|$ are dominated by $\max \{|v|,|w|\}$. Hence by the dominated convergence theorem, $\underline{u}, \bar{u} \in L^{1}\left(Q_{T}\right)$ and

$$
\lim _{k \rightarrow \infty} \int_{0}^{t} \int_{\mathbb{R}^{n}} G(x-y, s) v_{k}(y, s) \mathrm{d} y \mathrm{~d} s=\int_{0}^{t} \int_{\mathbb{R}^{n}} G(x-y, s) \underline{u}(y, s) \mathrm{d} y \mathrm{~d} s
$$

and

$$
\lim _{k \rightarrow \infty} \int_{0}^{t} \int_{\mathbb{R}^{n}} G(x-y, s) w_{k}(y, s) \mathrm{d} y \mathrm{~d} s=\int_{0}^{t} \int_{\mathbb{R}^{n}} G(x-y, s) \bar{u}(y, s) \mathrm{d} y \mathrm{~d} s .
$$

It follows that $\underline{u}$ and $\bar{u}$ are both integral solutions of (1.1), i.e. satisfy

$$
u(t)=S(t) \phi+\int_{0}^{t} S(t-s) f(u(s)) \mathrm{d} s
$$

a.e. in $Q_{T}$. Furthermore, by the integral condition (I1) we have that

$$
\begin{aligned}
\int_{0}^{T}\|f(v(s))\|_{1} \mathrm{~d} s & \leq \int_{0}^{T} \ell\left(2\left\|\phi^{-}\right\|_{1} s^{-n / 2}\right)\left\|2 S(s) \phi^{-}\right\|_{1} \mathrm{~d} s \\
& \leq 2\|\phi\|_{1} \int_{0}^{T} \ell\left(2\|\phi\|_{1} s^{-n / 2}\right) \mathrm{d} s<\infty
\end{aligned}
$$

and

$$
\begin{aligned}
\int_{0}^{T}\|f(w(s))\|_{1} \mathrm{~d} s & \leq \int_{0}^{T} \ell\left(2 s^{-n / 2}\left\|\phi^{+}\right\|_{1}\right)\left\|2 S(s) \phi^{+}\right\|_{1} \mathrm{~d} s \\
& \leq 2\|\phi\|_{1} \int_{0}^{T} \ell\left(2\|\phi\|_{1} s^{-n / 2}\right) \mathrm{d} s<\infty .
\end{aligned}
$$

Hence $f(\bar{u}) \in L^{1}\left((0, T), L^{1}\left(\mathbb{R}^{n}\right)\right)$ since $f(v) \leq f(\bar{u}) \leq f(w)$. It follows that $\bar{u} \in C\left([0, T], L^{1}\left(\mathbb{R}^{n}\right)\right)$ by (2.11). Furthermore, since $v, w \in L_{\text {loc }}^{\infty}\left((0, T), L^{\infty}\left(\mathbb{R}^{n}\right)\right)$ we also have $\bar{u} \in L_{\text {loc }}^{\infty}\left((0, T), L^{\infty}\left(\mathbb{R}^{n}\right)\right)$. 
As $f$ is Lipschitz continuous, standard parabolic regularity theory now implies that $\bar{u} \in$ $C^{2,1}\left(Q_{T}\right)$ and is a classical solution of (1.1). Thus, $\bar{u}$ is a classical $L^{1}$-solution of (1.1) on $[0, T)$.

By exactly the same argument we also deduce that $\underline{u}$ is a classical $L^{1}$-solution of (1.1) on $[0, T)$, with $\underline{u} \leq \bar{u}$.

That $t^{n / 2}\|\bar{u}(t ; \phi)\|_{\infty} \rightarrow 0$ and $t^{n / 2}\|\underline{u}(t ; \phi)\|_{\infty} \rightarrow 0$ as $t \rightarrow 0$ follows easily from the ordering

$$
2 S(t) \phi^{-} \leq \underline{u}(t ; \phi) \leq \bar{u}(t ; \phi) \leq 2 S(t) \phi^{+}
$$

and Lemma 2.1 with $q=1$ and $r=\infty$.

Remark 2.2. For notational convenience, in all that follows we simply write $T_{\phi}$ for $T_{\{\phi\}}$ when $B=\{\phi\}$.

\subsection{Uniqueness}

The proof of Theorem 2.2 provides a particular method for constructing ordered classical $L^{1}$-solutions $\underline{u}$ and $\bar{u}$ of (1.1) via a monotone iteration scheme. Under a stronger integral condition than (I1), involving the modulus of continuity of $f$, we will show that $\bar{u}$ is unique among all classical $L^{1}$-solutions of (1.1).

For $f$ satisfying $(\mathbf{M})$, define the non-decreasing function $L:[0, \infty) \rightarrow[0, \infty)$ by

$$
L(s)=\sup _{\substack{|u|,|v| \leq s, u \neq v}} \frac{f(u)-f(v)}{u-v} \quad(s>0), \quad L(0)=0 .
$$

We introduce the integral condition

(I2) $\quad \int_{1}^{\infty} s^{-p_{F}} L(s) \mathrm{d} s<\infty$.

Remark 2.3. Observe that $L(s) \geq \ell(s)$ and so (I2) implies (I1). We also note that (I2) is equivalent to

$$
\int_{0}^{1} L\left(\tau^{-n / 2}\right) \mathrm{d} \tau<\infty
$$


Proposition 2.3. Let $\phi, \psi \in L^{1}\left(\mathbb{R}^{n}\right), B=\{\phi, \psi\}$ and suppose that $f$ satisfies (M) and (I2). Let $\bar{u}(t ; \cdot)$ and $T_{B}$ be as in Theorem 2.2. Then there exists $\tau=\tau(\phi, \psi)>0$ and a continuous, non-negative function $q(t)$ satisfying $q(t) \rightarrow 0$ as $t \rightarrow 0$, such that for all $t \in(0, \tau]$

$$
\|\bar{u}(t ; \phi)-\bar{u}(t ; \psi)\|_{1}+t^{n / 2}\|\bar{u}(t ; \phi)-\bar{u}(t ; \psi)\|_{\infty} \leq 2\|\phi-\psi\|_{1} \mathrm{e}^{q(t)}
$$

Proof. To simplify notation let $u(t)=\bar{u}(t ; \phi)$ and $v(t)=\bar{u}(t ; \psi)$. By Theorem 2.2 there exists $\tau=\tau(\phi, \psi) \in\left(0, T_{B}\right)$ such that

$$
\|u(t)\|_{\infty} \leq t^{-n / 2} \quad \text { and } \quad\|v(t)\|_{\infty} \leq t^{-n / 2}
$$

for all $t \in(0, \tau]$. For such $t$,

$$
\begin{aligned}
\|u(t)-v(t)\|_{1} & \leq\|S(t)(\phi-\psi)\|_{1}+\int_{0}^{t}\|S(t-s)(f(u(s))-f(v(s)))\|_{1} \mathrm{~d} s \\
& \leq\|\phi-\psi\|_{1}+\int_{0}^{t}\left\|\frac{f(u(s))-f(v(s))}{u(s)-v(s)}\right\|_{\infty}\|S(t-s)(u(s)-v(s))\|_{1} \mathrm{~d} s \\
& \leq\|\phi-\psi\|_{1}+\int_{0}^{t} L\left(s^{-n / 2}\right)\|u(s)-v(s)\|_{1} \mathrm{~d} s
\end{aligned}
$$

by (2.12 2.14). Next, we have

$$
\begin{aligned}
\|u(t)-v(t)\|_{\infty} \leq & \|S(t)(\phi-\psi)\|_{\infty}+\int_{0}^{t}\|S(t-s)(f(u(s))-f(v(s)))\|_{\infty} \mathrm{d} s \\
& \leq t^{-n / 2}\|\phi-\psi\|_{1}+\int_{t / 2}^{t}\|f(u(s))-f(v(s))\|_{\infty} \mathrm{d} s \\
& +\int_{0}^{t / 2}\left\|\frac{f(u(s))-f(v(s))}{u(s)-v(s)}\right\|_{\infty}\|S(t-s)(u(s)-v(s))\|_{\infty} \mathrm{d} s \\
& \leq t^{-n / 2}\|\phi-\psi\|_{1}+\int_{t / 2}^{t} L\left(s^{-n / 2}\right)\|u(s)-v(s)\|_{\infty} \mathrm{d} s \\
& +\int_{0}^{t / 2} L\left(s^{-n / 2}\right)(t-s)^{-n / 2}\|u(s)-v(s)\|_{1} \mathrm{~d} s \\
& \leq t^{-n / 2}\|\phi-\psi\|_{1}+(t / 2)^{-n / 2} \int_{t / 2}^{t} L\left(s^{-n / 2}\right) s^{n / 2}\|u(s)-v(s)\|_{\infty} \mathrm{d} s
\end{aligned}
$$




$$
\begin{aligned}
& +(t / 2)^{-n / 2} \int_{0}^{t / 2} L\left(s^{-n / 2}\right)\|u(s)-v(s)\|_{1} \mathrm{~d} s \\
& \leq t^{-n / 2}\|\phi-\psi\|_{1}+(t / 2)^{-n / 2} \int_{0}^{t} L\left(s^{-n / 2}\right) s^{n / 2}\|u(s)-v(s)\|_{\infty} \mathrm{d} s \\
& +(t / 2)^{-n / 2} \int_{0}^{t} L\left(s^{-n / 2}\right)\|u(s)-v(s)\|_{1} \mathrm{~d} s .
\end{aligned}
$$

Combining (2.15) 2.16) we obtain, for all $t \in(0, \tau]$,

$$
\begin{aligned}
\|u(t)-v(t)\|_{1} & +t^{n / 2}\|u(t)-v(t)\|_{\infty} \leq 2\|\phi-\psi\|_{1} \\
& +\int_{0}^{t} k_{n} L\left(s^{-n / 2}\right)\left(\|u(s)-v(s)\|_{1}+s^{n / 2}\|u(s)-v(s)\|_{\infty}\right) \mathrm{d} s,
\end{aligned}
$$

where $k_{n}=\left(1+2^{n / 2}\right)$.

Now define $y(t)$ on $[0, \tau]$ by $y(0)=\|\phi-\psi\|_{1}$ and

$$
y(t)=\|u(t)-v(t)\|_{1}+t^{n / 2}\|u(t)-v(t)\|_{\infty}, \quad t \in(0, \tau] .
$$

By assumption $u, v \in C\left([0, \tau], L^{1}\left(\mathbb{R}^{n}\right)\right)$ and are both classical solutions for $t>0$. By standard parabolic regularity results we have that $u, v \in C\left((0, \tau), L^{\infty}\left(\mathbb{R}^{n}\right)\right)$, so that $y$ is continuous on $(0, \tau]$. By Theorem 2.2 it follows that $y$ is continuous on $[0, \tau]$. Hence by (2.17), (I2) and the singular Gronwall inequality (see e.g., [16, Ch.XII, Theorem 4]), it follows that

$$
\|u(t)-v(t)\|_{1}+t^{n / 2}\|u(t)-v(t)\|_{\infty} \leq 2\|\phi-\psi\|_{1} \mathrm{e}^{q(t)}
$$

for all $t \in(0, \tau]$, where

$$
q(t)=k_{n} \int_{0}^{t} L\left(s^{-n / 2}\right) \mathrm{d} s .
$$

Clearly $q$ is continuous with $q(t) \rightarrow 0$ as $t \rightarrow 0$ by (I2) (recalling (2.13) ), and the proof is complete.

We are now in a position to establish our main uniqueness result.

Theorem 2.4. (Uniqueness.) Let $\phi \in L^{1}\left(\mathbb{R}^{n}\right)$ and suppose that $f$ satisfies (M) and (I2). Let $\bar{u}(t ; \phi)$ and $T_{\phi}$ be as in Theorem 2.2. Then for any $T \leq T_{\phi}, \bar{u}(t ; \phi)$ is the unique classical $L^{1}$-solution of (1.1) on $[0, T)$. 
Proof. Let $u(t):=\bar{u}(t ; \phi)$ for $t \in\left[0, T_{\phi}\right)$ and $T \leq T_{\phi}$ be arbitrary. Suppose there exists a classical $L^{1}$-solution $v$ on $[0, T)$ with $v(0)=\phi$. By classical $L^{\infty}$-theory, it is clear that if there exists a $\tau \in(0, T)$ such that $u(t)=v(t)$ on $(0, \tau]$, then $u(t)=v(t)$ on $[0, T)$, i.e. uniqueness for sufficiently small times implies uniqueness on $[0, T)$.

Fix $\tau \in(0, T)$ and let $B=v([0, \tau])$. Since $v \in C\left([0, \tau], L^{1}\left(\mathbb{R}^{n}\right)\right), B$ is a bounded subset of $L^{1}\left(\mathbb{R}^{n}\right)$. By Theorem 2.2 there exists a $T_{B} \in\left(0, T_{\phi}\right]$ such that $\bar{u}(t ; \psi)$ is well-defined for all $\psi \in B$ and $t \in\left[0, T_{B}\right)$. Hence for all $t \in\left[0, T_{B}\right)$ we may define $U_{B}(t): B \rightarrow L^{1}\left(\mathbb{R}^{n}\right)$ by $U_{B}(t) \psi=\bar{u}(t ; \psi)$ and deduce from Proposition 2.3 that $U_{B}(t)$ is continuous (with respect to the induced $L^{1}$-norm). Again by classical $L^{\infty}$-uniqueness theory we have that $v(t+s)=$ $U_{B}(t) v(s)$ for all $t \geq 0$ sufficiently small and $s>0$ sufficiently small. Letting $s \rightarrow 0$, and using the continuity of $v:[0, \tau] \rightarrow L^{1}\left(\mathbb{R}^{n}\right)$ and $U_{B}(t): B \rightarrow L^{1}\left(\mathbb{R}^{n}\right)$, we therefore obtain $v(t)=U_{B}(t) v(0)=U_{B}(t) \phi=u(t)$ for all sufficiently small $t>0$, as required.

By Theorem 2.4 we may now dispense with the overbar (underbar) notation and simply write $u$ for $\bar{u}$ (and $\underline{u}$ ) whenever $\phi \in L^{1}\left(\mathbb{R}^{n}\right)$ and $f$ satisfies (M) and (I2).

\subsection{Continuous Dependence and Comparison}

As a consequence of Theorem 2.2 and Theorem 2.4 we may now deduce the existence of a maximally continued solution and obtain the usual blow-up alternative on its maximal interval of existence.

Theorem 2.5. (Maximal Solution.) Let $\phi \in L^{1}\left(\mathbb{R}^{n}\right)$ and suppose that $f$ satisfies (M) and (I2). Let $u(t ; \phi)$ denote the unique classical $L^{1}$-solution on $\left[0, T_{\phi}\right)$. Then there exists $T_{\max }(\phi) \geq T_{\phi}$ such that:

(a) $u(t ; \phi)$ can be continued (in a unique way) to a classical $L^{1}$-solution on $\left[0, T_{\max }(\phi)\right)$;

(b) if $T_{\max }(\phi)<\infty$ then $u(t ; \phi)$ cannot be continued to a classical $L^{1}$-solution on $[0, \tau)$ for any $\tau>T_{\max }(\phi)$;

(c) if $T_{\max }(\phi)<\infty$ then $\|u(t ; \phi)\|_{1} \rightarrow \infty$ as $t \rightarrow T_{\max }(\phi)$. 
Proof. Follows in the standard way (see [19, Proposition 16.1] for example) by the uniform existence result of Theorem 2.2 and uniqueness of Theorem 2.4 .

Part (a) of the following theorem is an immediate consequence of Proposition 2.3 and Theorem 2.4. We choose to state it explicitly in order to summarize our results more clearly but especially because Proposition 2.3 refers only to solutions obtained via the monotone iteration scheme, as opposed to any classical $L^{1}$-solution.

Theorem 2.6. Let $\phi, \psi \in L^{1}\left(\mathbb{R}^{n}\right)$ and suppose that $f$ satisfies $(\mathbf{M})$ and $(\mathbf{I} 2)$. Let $u(t ; \phi)$ and $u(t ; \psi)$ be as in Theorem 2.5.

(a) (Continuous Dependence.) There exists $\tau=\tau(\phi, \psi)>0$ and a continuous, nonnegative function $q(t)$ satisfying $q(t) \rightarrow 0$ as $t \rightarrow 0$, such that for all $t \in(0, \tau]$,

$$
\|u(t ; \phi)-u(t ; \psi)\|_{1}+t^{n / 2}\|u(t ; \phi)-u(t ; \psi)\|_{\infty} \leq 2\|\phi-\psi\|_{1} \mathrm{e}^{q(t)} .
$$

(b) (Comparison.) If $\phi \leq \psi$ then $u(t ; \phi) \leq u(t ; \psi)$ on $[0, T)$, for all $T \leq \min \left\{T_{\max }(\phi), T_{\max }(\psi)\right\}$. In particular, if $\phi \geq 0$ then $u(t ; \phi) \geq 0$ on $\left[0, T_{\max }(\phi)\right)$.

Proof. For part (b) we recall from Theorem 2.2 and Theorem 2.4 that there exists a $T_{\phi} \leq$ $T_{\max }(\phi)$ such that the classical $L^{1}$-solution $u(t ; \phi)$ is obtained as the pointwise limit of the iteration scheme

$$
w_{k+1}(t ; \phi)=\mathscr{F}\left(w_{k} ; \phi\right), \quad w_{0}(t ; \phi)=2 S(t) \phi^{+}
$$

for $t \in\left[0, T_{\phi}\right)$.

Similarly we obtain the classical $L^{1}$-solution $u(t ; \psi)$ on $\left[0, T_{\psi}\right)$ via

$$
w_{k+1}(t ; \psi)=\mathscr{F}\left(w_{k} ; \psi\right), \quad w_{0}(t ; \psi)=2 S(t) \psi^{+} .
$$

Let $T_{0}=\min \left\{T_{\phi}, T_{\psi}\right\}$. Clearly

$$
w_{0}(t ; \phi)=2 S(t) \phi^{+} \leq 2 S(t) \psi^{+}=w_{0}(t ; \psi),
$$


and since the operator $\mathscr{F}$ is order-preserving in both its arguments (due to monotonicity of $f$ and $S(t))$, it follows easily by induction that $w_{k}(t ; \phi) \leq w_{k}(t ; \psi)$ for all $k$. Letting $k \rightarrow \infty$ we therefore have $u(t ; \phi) \leq u(t ; \psi)$ on $\left[0, T_{0}\right)$. The comparison result on $[0, T)$ for any $T \leq \max \left\{T_{\max }(\phi), T_{\max }(\psi)\right\}$ now follows by standard $L^{\infty}$-comparison results for classical solutions since $f$ is locally Lipschitz continuous.

The final part of (b) follows by comparison and the fact that $u(t ; 0)=0$ for all $t \geq 0$ by uniqueness.

\subsection{Global Solutions}

Next we establish some sufficient conditions for the global continuation of solutions with small intial data. The following integral condition plays the key rôle:

(I3) $\quad \int_{0}^{1} s^{-p_{F}} \ell(s) \mathrm{d} s<\infty$.

Theorem 2.7. Suppose $\phi \in L^{1}\left(\mathbb{R}^{n}\right) \cap L^{\infty}\left(\mathbb{R}^{n}\right)$ and $(\mathbf{M})$ holds. Let $u_{c}(t ; \phi)$ denote the unique classical $L^{\infty}$-solution of (1.1) with maximal (in $L^{\infty}$ ) interval of existence $\left[0, T_{\max }(\phi)\right)$. If (I3) holds, then for any $A>1$ there exists $\delta=\delta(A)>0$ such that for all $\phi$ satisfying $\|\phi\|_{1}+\|\phi\|_{\infty} \leq \delta$ we have $T_{\max }(\phi)=\infty$ and

$$
A S(t) \phi^{-} \leq u_{c}(t ; \phi) \leq A S(t) \phi^{+}
$$

for all $t \geq 0$. Consequently $\left\|u_{c}(t ; \phi)\right\|_{\infty} \leq A t^{-n / 2}\|\phi\|_{1}$ for all $t>0$.

Proof. First, we will show that for fixed $A>1$ and suitably small $\delta>0, v:=A S(t) \phi^{-}$and $w:=A S(t) \phi^{+}$are an integral sub-supersolution pair for (1.1) for all $t \geq 0$. Similarly to Theorem 2.2 we will then be able to deduce the existence of a pair of classical $L^{\infty}$-solutions $\underline{u}$ and $\bar{u}$ satisfying

$$
v \leq \underline{u} \leq \bar{u} \leq w
$$

By uniqueness of classical $L^{\infty}$-solutions (see e.g., [19, Section 51, Appendix E]) we may then conclude that $\underline{u}=\bar{u}=u_{c}$ and hence $v \leq u_{c}(t ; \phi) \leq w$, yielding (2.18). The $L^{\infty}$ bound for $u_{c}$ then follows by $L^{1}-L^{\infty}$ smoothing via Lemma 2.1. It is therefore sufficient to prove the existence of solutions $\underline{u}$ and $\bar{u}$ satisfying (2.19). 
Let $\delta \leq 1$ and choose $\tau>0$ such that $1-A+A \ell(A) \tau<0$. By (2.2) with $q=r=\infty$ we have $\|S(t) \phi\|_{\infty} \leq\|\phi\|_{\infty} \leq \delta \leq 1$ for all $t \geq 0$. In particular, for all $t \in[0, \tau]$ we have

$$
\begin{aligned}
\mathscr{F}(w ; \phi)-w & =S(t) \phi+\int_{0}^{t} S(t-s) f(w(s)) \mathrm{d} s-w \\
& \leq S(t) \phi^{+}+\int_{0}^{t} S(t-s)[\ell(w(s)) w(s)] \mathrm{d} s-w \\
& =(1-A) S(t) \phi^{+}+\int_{0}^{t} S(t-s)\left[\ell\left(A S(s) \phi^{+}\right) A S(s) \phi^{+}\right] \mathrm{d} s \\
& \leq(1-A) S(t) \phi^{+}+\int_{0}^{t} S(t-s)\left[\ell\left(\left\|A S(s) \phi^{+}\right\|_{\infty}\right) A S(s) \phi^{+}\right] \mathrm{d} s \\
& \leq(1-A) S(t) \phi^{+}+\int_{0}^{t} S(t-s)\left[\ell(A) A S(s) \phi^{+}\right] \mathrm{d} s \\
& =(1-A) S(t) \phi^{+}+A \ell(A) \int_{0}^{t} S(t) \phi^{+} \mathrm{d} s \\
& \leq(1-A+A \ell(A) \tau) S(t) \phi^{+} \leq 0 .
\end{aligned}
$$

For $t>\tau$ we proceed as above, again making use of $L^{1}-L^{\infty}$ smoothing:

$$
\begin{aligned}
& \mathscr{F}(w ; \phi)-w=S(t) \phi+\int_{0}^{\tau} S(t-s) f(w(s)) \mathrm{d} s+\int_{\tau}^{t} S(t-s) f(w(s)) \mathrm{d} s-w \\
\leq & (1-A+A \ell(A) \tau) S(t) \phi^{+}+\int_{\tau}^{t} S(t-s)[\ell(w(s)) w(s)] \mathrm{d} s \\
\leq & (1-A+A \ell(A) \tau) S(t) \phi^{+}+\int_{\tau}^{t} S(t-s)\left[\ell\left(\left\|A S(s) \phi^{+}\right\|_{\infty}\right) A S(s) \phi^{+}\right] \mathrm{d} s \\
\leq & (1-A+A \ell(A) \tau) S(t) \phi^{+}+\int_{\tau}^{t} S(t-s)\left[\ell\left(A \delta s^{-n / 2}\right) A S(s) \phi^{+}\right] \mathrm{d} s \\
= & (1-A+A \ell(A) \tau) S(t) \phi^{+}+A S(t) \phi^{+} \int_{\tau}^{t} \ell\left(A \delta s^{-n / 2}\right) \mathrm{d} s \\
= & \left(1-A+A \ell(A) \tau+(2 / n) A^{p_{F}} \delta^{2 / n} \int_{A \delta t^{-n / 2}}^{A \delta \tau^{-n / 2}} z^{-p_{F}} \ell(z) \mathrm{d} z\right) S(t) \phi^{+} \\
\leq & \left(1-A+A \ell(A) \tau+(2 / n) A^{p_{F}} \delta^{2 / n} \int_{0}^{A \delta \tau^{-n / 2}} z^{-p_{F}} \ell(z) \mathrm{d} z\right) S(t) \phi^{+} \\
\leq & 0
\end{aligned}
$$

for $\delta$ sufficiently small (and independently of $t$ ), by (I3).

In exactly the same way (and similar to that in the proof of Theorem 2.2 - see the calculations surrounding (2.8) ) one may verify that $v$ is an integral subsolution. We omit the 
repetitive details. Likewise one can again construct monotonic sequences $v_{k}$ and $w_{k}$ as in (2.9) 2.10), converging pointwise to limiting functions $\underline{u}$ and $\bar{u}$ respectively, with $v \leq \underline{u} \leq \bar{u} \leq w$. Via the properties shared by $v$ and $w$ and the dominated convergence theorem, $\underline{u}$ and $\bar{u}$ both satisfy the variation of constants formula (2.1) almost everywhere in $Q_{T}$, for any $T>0$. Furthermore $\underline{u}$ and $\bar{u}$ are both essentially bounded in $Q_{T}$ and consequently they are classical solutions of (1.1). This completes the proof.

For initial data which are not necessarily bounded we also require $f$ to satisfy the integral condition (I2).

Theorem 2.8. Suppose $\phi \in L^{1}\left(\mathbb{R}^{n}\right)$ and $(\mathbf{M})$ and (I2) hold. Let $u(t ; \phi)$ denote the unique classical $L^{1}$-solution of (1.1) guaranteed by Theorem 2.5, with maximal interval of existence $\left[0, T_{\max }(\phi)\right)$. If (I3) holds, then for any $A>1$ there exists $\delta=\delta(A)>0$ such that for all $\phi$ satisfying $\|\phi\|_{1} \leq \delta$ we have $T_{\max }(\phi)=\infty$ and

$$
A S(t) \phi^{-} \leq u(t ; \phi) \leq A S(t) \phi^{+}
$$

for all $t \geq 0$. Consequently $\|u(t ; \phi)\|_{\infty} \leq A t^{-n / 2}\|\phi\|_{1}$ for all $t>0$.

Proof. It is easily verified that $v=A S(t) \phi^{-}$and $w=A S(t) \phi^{+}$are a pair of global integral sub-supersolutions by simply taking $\tau=0$ in the calculations in the proof of Theorem 2.7 , for example,

$$
\mathscr{F}(w ; \phi)-w \leq\left(1-A+(2 / n) A^{p_{F}} \delta^{2 / n} \int_{0}^{\infty} z^{-p_{F}} \ell(z) \mathrm{d} z\right) S(t) \phi^{+} \leq 0
$$

for $\delta$ sufficiently small by (I2) (see Remark 2.3) and (I3). Similarly for $v$.

The usual monotone iteration procedure then yields a pair of global classical $L^{1}$-solutions $\underline{u}$ and $\bar{u}$ satisfying $v \leq \underline{u} \leq \bar{u} \leq w$ for all $t \geq 0$. By the uniqueness result of Theorem 2.4 it follows that $\underline{u}=\bar{u}=u(t ; \phi)$ on $\left[0, T_{\max }(\phi)\right)$ and so $T_{\max }(\phi)=\infty$. The $L^{\infty}$ bound for $u$ follows once more by the ordering $A S(t) \phi^{-} \leq u \leq A S(t) \phi^{+}$and $L^{1}-L^{\infty}$ smoothing of the heat semigroup. 
Example 2.1. Let $1<p<p_{F}<q$ and $f_{+}:[0, \infty) \rightarrow[0, \infty)$ be the non-convex, locally Lipschitz function

$$
f_{+}(u)=\min \left\{u^{p}, u^{q}\right\}
$$

so that $f_{+}(u)=u^{q}$ for $0 \leq u \leq 1$ and $f_{+}(u)=u^{p}$ for $u>1$. Let $f: \mathbb{R} \rightarrow \mathbb{R}$ be the odd extension of $f_{+}$. Then $f$ satisfies the hypotheses of both Theorem 2.5 and Theorem 2.8 , with $L(s)=p s^{p-1}$ for $s$ large and $\ell(s)=s^{q-1}$ for $s$ small. This provides an example of a source term $f$ for which (1.1) is both well-posed in $L^{1}\left(\mathbb{R}^{n}\right)$ and possesses non-trivial global solutions, in contrast to the homogeneous power law case $f(u)=|u|^{p-1} u, p>1$.

\section{Special Cases}

\subsection{Convex Source Terms}

We now show that the 'gap' between the integral condition (I1) for existence and the one for uniqueness, (I2), vanishes when the source term $f$ is odd and is convex on $(0, \infty)$.

Lemma 3.1. Assume (M) and (I1) hold. If $f$ is odd and is convex on $(0, \infty)$, then $f$ satisfies (I2).

Proof. Since $f$ is locally Lipschitz it is differentiable a.e., with derivative $f^{\prime}$ at all such points. The oddness of $f$ together with its convexity on $(0, \infty)$ then imply that $\ell(s)=f(s) / s$ and $L(s)=f^{\prime}(s)$ a.e. Hence,

$$
\begin{aligned}
\int_{1}^{\infty} s^{-p_{F}} L(s) \mathrm{d} s & =\int_{1}^{\infty} s^{-p_{F}} f^{\prime}(s) \mathrm{d} s \\
& =\left[s^{-p_{F}} f(s)\right]_{1}^{\infty}+p_{F} \int_{1}^{\infty} s^{-p_{F}} \ell(s) \mathrm{d} s
\end{aligned}
$$

The latter integral in (3.1) is finite by (I1). Also,

$$
\int_{s}^{2 s} t^{-p_{F}} \ell(t) \mathrm{d} t \geq \ell(s) \int_{s}^{2 s} t^{-p_{F}} \mathrm{~d} t=C_{n} s^{-p_{F}} f(s)
$$

and so by (I1) $s^{-p_{F}} f(s) \rightarrow 0$ as $s \rightarrow \infty$. Consequently the right hand side of (3.1) is finite and (I2) holds. 
Corollary 3.2. Assume $f$ is odd, convex on $(0, \infty)$ and satisfies $(\mathbf{M})$. If $\int_{1}^{\infty} s^{-(2+2 / n)} f(s) \mathrm{d} s<$ $\infty$ then the conclusions of Theorem 2.5 and Theorem 2.6 hold.

Example 3.1. In the special case of the homogeneous power law $f(u)=|u|^{p-1} u, p>1$, Corollary 3.2 is applicable if and only if $p<p_{F}$. See for example [3, 22, 23].

One can also obtain a result like Corollary 3.2 without requiring $f$ to be odd, provided that one redefines (I1) and (I2) accordingly. For example, if $f$ is concave on $(-\infty, 0)$ and convex on $(0, \infty)$, then $\ell(s)=\max \{f(s) / s,-f(-s) / s\}$ and $L(s)=\max \left\{f^{\prime}(s),-f^{\prime}(-s)\right\}$ a.e. One then replaces (I1) by the pair of integral conditions

$$
\int_{1}^{\infty} s^{-(2+2 / n)} f(s) \mathrm{d} s<\infty \text { and } \quad-\int_{-\infty}^{-1}(-s)^{-(2+2 / n)} f(s) \mathrm{d} s<\infty
$$

and analogously for (I2).

Assuming (M) holds we have shown that (I2) is sufficient for the well-posedness of (1.1) in $L^{1}\left(\mathbb{R}^{n}\right)$, together with comparison and continuous dependence of solutions. It is natural therefore to ask whether (I2) is necessary for well-posedness. We have a partial result in this direction.

Corollary 3.3. Assume $f$ is odd, convex on $(0, \infty)$ and satisfies $(\mathbf{M})$. If $f$ does not satisfy (I2) then either (1.1) is not well-posed in $L^{1}\left(\mathbb{R}^{n}\right)$ or the comparison principle fails.

Proof. By Lemma 3.1, if $f$ does not satisfy (I2) then does not satisfy (I1). Therefore, by [13, Theorem 5.1] there exists a non-negative initial condition $\phi \in L^{1}\left(\mathbb{R}^{n}\right)$ such that (1.1) does not possess a local non-negative integral solution (and hence no non-negative classical $L^{1}$-solution). It follows that if (1.1) is well-posed in $L^{1}\left(\mathbb{R}^{n}\right)$ then the corresponding unique classical $L^{1}$-solution $u(t ; \phi)$ must be sign-changing on every small time interval $(0, T)$. Comparison with $u(t ; 0)=0$ consequently fails.

Recalling Corollary 3.2 we may combine the results of Theorem 2.5 and Theorem 2.8 in the following special case. 
Corollary 3.4. Suppose $f$ is odd, convex on $(0, \infty)$ and satisfies (M). If

$$
\int_{0}^{\infty} \frac{f(s)}{s^{2+2 / n}} \mathrm{~d} s<\infty
$$

then (1.1) is well-posed in $L^{1}\left(\mathbb{R}^{n}\right)$ and all classical $L^{1}$-solutions having sufficiently small initial data in $L^{1}\left(\mathbb{R}^{n}\right)$ are global in time, decaying uniformly to zero like $O\left(t^{-n / 2}\right)$ as $t \rightarrow \infty$.

\subsection{Positive Solutions}

Here we outline some consequences relating specifically to non-negative solutions. The results of previous sections are easily paralleled by replacing $L^{1}\left(\mathbb{R}^{n}\right)$ throughout by $L_{+}^{1}\left(\mathbb{R}^{n}\right)$, the cone of non-negative functions in $L^{1}\left(\mathbb{R}^{n}\right)$. In particular the definitions of solution and well-posedness in Definition 2.1 are now made with respect to $L_{+}^{1}\left(\mathbb{R}^{n}\right)$ rather than $L^{1}\left(\mathbb{R}^{n}\right)$, with $\phi \in L_{+}^{1}\left(\mathbb{R}^{n}\right)$.

First we replace $(\mathbf{M})$ by

$(\mathbf{M})_{+} \quad f:[0, \infty) \rightarrow[0, \infty)$ is locally Lipschitz continuous, non-decreasing and $f(0)=0$, and (2.3) by

$$
\ell_{+}(s)=\sup _{0<t \leq s} \frac{f(t)}{t} \quad(s>0), \quad \ell_{+}(0)=0 .
$$

We then replace $\ell$ by $\ell_{+}$in the integral condition (I1):

$$
(\mathrm{I} 1)_{+} \int_{1}^{\infty} s^{-p_{F}} \ell_{+}(s) \mathrm{d} s<\infty .
$$

The function $\underline{u}$ in Theorem 2.2 is then obtained by monotone iteration of the integral subsolution $v=A S(t) \phi^{-}=0$, since now $\phi^{-}=0$. Likewise we replace $L$ in (2.12) by

$$
L_{+}(s)=\sup _{\substack{0<u, v \leq s, u \neq v}} \frac{f(u)-f(v)}{u-v} \quad(s>0), \quad L_{+}(0)=0
$$

and $L$ by $L_{+}$in (I2):

$$
(\mathbf{I} 2)_{+} \quad \int_{1}^{\infty} s^{-p_{F}} L_{+}(s) \mathrm{d} s<\infty
$$


One readily deduces that if $f$ satisfies $(\mathbf{M})_{+}$and $(\mathbf{I} 2)_{+}$then for every $\phi \in L_{+}^{1}\left(\mathbb{R}^{n}\right)$ there exists a $T_{\max }(\phi)>0$ and a unique, non-negative classical $L^{1}$-solution $u(t ; \phi)$ on $\left[0, T_{\max }(\phi)\right)$. Furthermore, if $T_{\max }(\phi)<\infty$ then $\|u(t ; \phi)\|_{1} \rightarrow \infty$ as $t \rightarrow T_{\max }(\phi)$. Similarly one obtains continuous dependence and comparison of non-negative solutions. This yields the analogues of Theorem 2.5 and Theorem 2.6 in $L_{+}^{1}\left(\mathbb{R}^{n}\right)$.

The counterparts of Theorems 2.7 and 2.8 regarding non-negative global solutions satisfying $0 \leq u(t ; \phi) \leq A S(t) \phi$ also follow in exactly the same way, on replacing (I3) with

$$
\text { (I3) }+\int_{0}^{1} s^{-p_{F}} \ell_{+}(s) \mathrm{d} s<\infty .
$$

In fact the rôle of $(\mathbf{I 3})_{+}$is known to be important in determining whether positive classical solutions decay to zero or whether they 'grow-up' [12], and possibly blow-up.

Example 3.2. Let $f:[0, \infty) \rightarrow[0, \infty)$ be given by $f(0)=0$ and $f(u)=u^{p_{F}} g(u)$ for $u>0$, where

$$
g(u)= \begin{cases}{[\ln (1 / u)]^{-\gamma},} & 0<u<a \\ g_{0}(u), & a \leq u \leq b \\ {[\ln (\mathrm{e}+u)]^{-\beta}} & u>b\end{cases}
$$

$\gamma, \beta>1$ are fixed and $a, b>0$ will be chosen below. This example combines those of [13, Section 4.4] on local existence of $L^{1}$-solutions and [12, Example 5.1] on global existence of classical solutions, in order to illustrate some of our results.

Choosing $a$ sufficiently small, $b$ sufficiently large and $g_{0}$ as a monotonic interpolant, we can ensure that $f$ satisfies $(\mathbf{M})_{+}$and moreover that $\ell_{+}(u)=f(u) / u$ on $(0, a)$ and $L_{+}(u)=f^{\prime}(u)$ for $u>b$. The choice of $\beta>1$ and $\gamma>1$ then ensure that $(\mathbf{I} 2)_{+}$and $(\mathbf{I} 3)_{+}$ hold, respectively. Consequently (1.1) is well-posed in $L_{+}^{1}\left(\mathbb{R}^{n}\right)$ with continuous dependence upon initial conditions and the comparison principle also assured. Furthermore, solutions with sufficiently small initial data in $L_{+}^{1}\left(\mathbb{R}^{n}\right)$ exist globally in time.

We emphasise that the results available in [3, 22, 23] are not sufficiently sharp to be able to deduce the kind of well-posedness results that we obatin here for source terms such as this. 
Similarly to Lemma 3.1 it again follows that if $f$ satisfies $(\mathbf{M})_{+}$and $(\mathbf{I 1})_{+}$and is convex, then $f$ satisfies $(\mathbf{I} 2)_{+}$. We therefore have the following interesting result:

Corollary 3.5. Suppose $f$ satisfies $(\mathbf{M})_{+}$and is convex. Then (1.1) is well-posed in $L_{+}^{1}\left(\mathbb{R}^{n}\right)$ if and only if $\int_{1}^{\infty} s^{-(2+2 / n)} f(s) \mathrm{d} s<\infty$. Moreover, if $\int_{1}^{\infty} s^{-(2+2 / n)} f(s) \mathrm{d} s<\infty$ then continuous dependence and comparison also hold in the sense of Theorem 2.6.

Proof. If $\int_{1}^{\infty} s^{-(2+2 / n)} f(s) \mathrm{d} s=\infty$ then by [13, Theorem 5.1] there exists a $\phi \in L_{+}^{1}\left(\mathbb{R}^{n}\right)$ such that (1.1) does not possess a local non-negative integral solution (and hence no non-negative classical $L^{1}$-solution). Consequently (1.1) is not well-posed in $L_{+}^{1}\left(\mathbb{R}^{n}\right)$.

Conversely, if $\int_{1}^{\infty} s^{-(2+2 / n)} f(s) \mathrm{d} s<\infty$, then by Lemma 3.1. Theorem 2.5, Theorem 2.6 and the discussion above, (1.1) is well-posed in $L_{+}^{1}\left(\mathbb{R}^{n}\right)$ and enjoys the continuous dependence and comparison properties stated.

\section{Concluding remarks}

We have established new results on the local well-posedness and global continuation of classical $L^{1}$-solutions of semilinear heat equations, extending those of [3, 13, 22, 23, 24] under less restrictive growth conditions on the source term $f$. Furthermore, we have also obtained continuous dependence and comparison results in this more general setting. Here we discuss several extensions to our work which seem to us to be readily achievable. For expositional reasons we have chosen not to present the details here; instead we outline the necessary steps.

We have derived results only for the Cauchy problem on $\mathbb{R}^{n}$. However, our results also hold in $L^{1}(\Omega)$ for bounded domains $\Omega$ with homogeneous Dirichlet or Neumann boundary conditions. The proofs require only minor (but frequent) modifications, along the lines of those in [13], and following the sub-supersolution methods used here with appropriate changes to the heat semigroup to incorporate the boundary conditions. In fact, by following the same argument as in [3, Remark 7.2] one can obtain uniqueness of classical $L^{1}$-solutions in the larger class

$$
C\left([0, T], L^{1}(\Omega)\right) \cap L_{\mathrm{loc}}^{\infty}\left((0, T), L^{p_{F}}(\Omega)\right)
$$


under the same hypotheses as Theorem 2.4 ,

Our well-posedness results in $L^{1}\left(\mathbb{R}^{n}\right)$ also carry through with minor modification if one replaces the Laplacian operator by the fractional Laplacian and consider instead the problem

$$
u_{t}=-(-\Delta)^{\beta / 2} u+f(u), \quad u(0)=\phi \in L^{1}\left(\mathbb{R}^{n}\right)
$$

for $\beta \in(0,2]$. The rôle of the Fujita-type exponent $p_{F}$ is then replaced by $p_{F}(\beta)=1+\beta / n$. Local and global existence results then follow by comparison with $A S_{\beta}(t) \phi^{ \pm}(A>1)$, where $S_{\beta}(t)$ denotes the semigroup generated by the fractional Laplacian. An appropriate integral sub-supersolution existence theorem is easily obtained by adapting those in [20] and suitable monotonicity and smoothing properties are also available. Some work along these lines for local existence/non-existence of non-negative solutions can be found in [14]. One may then adapt our methods here to obtain the analogous uniqueness, continuous dependence and comparison results.

\section{Acknowledgements}

This work was partially supported under the Research in Pairs scheme at the Centre International de Rencontres Mathématiques, Luminy. MS was also partially supported by NCN grant 2017/26/D/ST1/00614. The authors would also like to thank the anonymous reviewers for their helpful suggestions to improve the final version of this manuscript.

\section{References}

[1] J. Arrieta and A. Carvalho. Abstract parabolic problems with critical nonlinearities and applications to Navier-Stokes and heat equations. Trans. Amer. Math. Soc. 352 (2000), 285-310.

[2] P. Baras and M. Pierre. Critère d'existence de solutions positives pour des équations semi-linéaires non monotones. Ann. Inst. H. Poincaré Anal. Non Linéaire, 2 (1985), 185-212.

[3] H. Brézis and T. Cazenave. A nonlinear heat equation with singular initial data. J. Anal. Math., 68 (1996), 277-304.

[4] C. Celik and Z. Zhou. No local $L^{1}$ solution for a nonlinear heat equation. Comm. Partial Differential Equations, 28 (2003), 1807-1831. 
[5] M. Fila, J.R. King, M. Winkler and E. Yanagida. Grow-up rate of solutions of a semilinear parabolic equation with a critical exponent. Adv. Differential Equations, 12 (2007), 1-26.

[6] S. Filippas, M.A. Herrero and J.J.L. Velázquez. Fast blow-up mechanisms for sign-changing solutions of a semilinear parabolic equation with critical nonlinearity. Proc. R. Soc. Lond. Ser. A Math. Phys. Eng. Sci., 456 (2000), 2957-2982.

[7] Y. Fujishima and N. Ioku. Existence and nonexistence of solutions for the heat equation with a superlinear source term. J. Math. Pures Appl., 118 (2018), 128-158.

[8] H. Fujita. On the blowing up of solutions of the Cauchy problem for $u_{t}=\Delta u+u^{1+\alpha}$. J. Sci. Univ. Tokyo, Sect. I, 13 (1966), 109-124.

[9] A. Haraux and F.B. Weissler. Non-uniqueness for a semilinear initial value problem. Indiana Univ. Math. J., 31 (1982), 167-189.

[10] K. Hayakawa. On nonexistence of global solutions of some semilinear parabolic differential equations. Proc. Japan Acad. Ser. A Math. Sci., 49 (1973), 503-505.

[11] S. Kaplan. On the growth of solutions of quasi-linear parabolic equations. Comm. Pure Appl. Math. 16 (1963), 305-330.

[12] K. Kobayashi, T. Sirao and H. Tanaka. On the growing up problem for semilinear heat equations. J. Math. Soc. Japan, 29 (3) (1977), 407-425.

[13] R. Laister, J.C. Robinson, M. Sierżęga and A. Vidal-López. A complete characterisation of local existence for semilinear parabolic equations in Lebesgue spaces. Ann. Inst. H. Poincaré Anal. Non Linéaire, 33 (6) (2016), 1519-1538.

[14] K. Li. A characteristic of local existence for nonlinear fractional heat equations in Lebesgue spaces. Comput. Math. Appl. 73 (2017), 653-665.

[15] J. Matos and E. Terraneo. Nonuniqueness for a critical nonlinear heat equation with any initial data. Nonlinear Analysis, 55 (2003), 927-936.

[16] D.S. Mitrinović, J. Pečarić and A.M. Fink. Inequalities Involving Functions and Their Integrals and Derivatives. Mathematics and its Applications. Kluwer Academic Publishers, Dordrecht, 1991.

[17] W-M. Ni and P. Sacks. Singular behavior in nonlinear parabolic equations. Trans. Amer. Math. Soc., 287 (1985), 657-671.

[18] P. Poláčik and E. Yanagida. On bounded and unbounded global solutions of a supercritical semilinear heat equation. Math. Ann., 327 (2003), 745-771.

[19] P. Quittner and P. Souplet. Superlinear Parabolic Problems. Blow-up, Global Existence and Steady States (2nd Edition). Birkhäuser Advanced Texts, Basel, 2019.

[20] J.C. Robinson and M. Sierżęa. Supersolutions for a class of semilinear heat equations. Rev. Mat. Complut., 26 (2013), 341-360. 
[21] S. Sugitani. On nonexistence of global solutions for some nonlinear integral equations. Osaka J. Math., $12(1975), 45-51$.

[22] F.B. Weissler. Semilinear evolution equations in Banach spaces. J. Funct. Anal., 32 (1979), 277-296.

[23] F.B. Weissler. Local existence and nonexistence for semilinear parabolic equations in $L^{p}$. Indiana Univ. Math. J., 29 (1980), 79-102.

[24] F.B. Weissler. Existence and nonexistence of global solutions for a semilinear heat equation. Israel J. Math., 38 (1981), 29-40. 